\title{
Ciel d'îles
}

\section{Marie-Françoise Peteuil}

\section{OpenEdition}

Journals

Édition électronique

URL : http://journals.openedition.org/jso/1142

DOI : 10.4000/jso. 1142

ISSN : 1760-7256

\section{Éditeur}

Société des océanistes

\section{Édition imprimée}

Date de publication : 1 juin 2003

Pagination : 13-24

ISSN : 0300-953x

\section{Référence électronique}

Marie-Françoise Peteuil, « Ciel d'îles », Journal de la Société des Océanistes [En ligne], 116 | Année 2003-1, mis en ligne le 26 mai 2008, consulté le 20 avril 2019. URL : http://journals.openedition.org/ jso/1142 ; DOl : 10.4000/jso.1142 


\section{Ciel d'îles}

par

Marie-Françoise PETEUIL *

\section{RÉSUMÉ}

Tout en soulignant les rapports existant en Polynésie entre la navigation et les étoiles (comme la boussole stellaire et l'etak), cet article tente de mettre en évidence d'autres liens, tissés entre les astres et les îles, les astres et les voyages, les astres et le temps, entre les noms des étoiles, les héros et la toponymie.

MoTS-CLÉS : Polynésie, étoiles, navigation hauturière, boussole stellaire, carte de Tupaia, etak, ethnoastronomie, mythologie polynésienne, toponymie.

$\mathrm{Au}$ moment des premiers contacts, les Européens réalisèrent très vite que les Polynésiens avaient une conscience claire de la dispersion de leurs îles, et de la petitesse de celles-ci sur le vaste océan. La carte des îles que Tupaia, le Tahitien qui fut l'informateur de Cook, dressa en 1778 pour celui-ci en témoigne. Ben Finney, en parle longuement (Finney, 1999: 100-104). On voit que Tupaia, prêtre et conseiller des grands chefs, se révéla être une mine de savoir géographique, météorologique et maritime. Il évoqua pour Cook les nombreuses îles entourant Tahiti, la manière de les atteindre, les longs voyages nécessaires. Il accepta de transcrire ses connaissances pour Cook, à la manière de Cook. Ce qui donna la fameuse « Carte de Tupaia », qui fut l'objet de nombreuses études, et se révéla très porteuse de sens. Cette carte s'étendait très loin, jusqu'aux îles Fidji, mais présentait des « erreurs » inexplicables. En 1838, Horatio Hale comprit qu'une

\section{ABSTRACT}

This paper focusses on the links found in Polynesia between navigation and the stars (such as the star compass and etak). It also attempts to show other links, such as those existing between stars and islands, stars and sea travels, stars and time, between the names of the stars, heroes and toponymy.

Keywords : Polynesia, stars, ocean navigation, star compass, Tupaia's map, etak, ethno-astronomy, Polynesian mythology, toponymy.

grande partie de ces erreurs venait de l'inversion du Nord et du Sud, notés Opatoarow et Opatoa sur le document, signifiant en fait «Vent du Nord » et « Vent du Sud ». Cook avait perçu ces termes selon le sens qu'on leur donne en Europe, vent qui vient du Nord, ou du Sud. Or, pour les Tahitiens, les vents sont nommés non pas selon la direction d'où ils viennent, mais selon la direction où ils poussent. Le vent «du Nord» pour Cook était celui qui pousse au Sud, donc vent « du Sud » pour les Tahitiens. Belle manière déjà de toucher la différence dans la façon géographique de se situer au monde: l'une statique qui perçoit le vent qui arrive, l'autre prête à se déplacer dès que se pointe l'opportunité d'une brise. Pas d'ambiguité pour l'Est et l'Ouest, le lever et le coucher du soleil étant des notions communes.

Alors que Cook et Tupaia étaient ainsi occupés à dresser cette fameuse carte, Johann Forster, le naturaliste de Cook, note : «Il [Tupaia] avait

* Professeur de mathématiques, astronome-amateur ; mariefpe@hotmail.com 
compris le rôle des cartes et donna ses instructions pour en obtenir une. Il pointait toujours $d u$ doigt les parties du ciel qui correspondaient aux iles [...] » (Finney, 1999 : 101)

Dans cette phrase, se découvre, me semble-t-il, tout un aspect de l'utilisation des étoiles par les Polynésiens qui a rarement été développé : le ciel nocturne compris comme une représentation de leur monde. Mon propos consistera à mettre en relief cette « lecture » des astres par les Polynésiens.

\section{Étoiles en verticalité}

Pour qui contemple la carte du Pacifique et sa poussière d'îles d'un certain œil, il y a une analogie certaine avec le ciel étoilé, même immensité bleue piquetée de petits points. Mais, contrairement à ce que l'on pourrait croire, la liaison que les Polynésiens entretenaient entre les deux n'était pas une comparaison immédiate, une superposition évidente, et très occidentale, entre carte d'océan et vision du ciel.

Dans la mythologie polynésienne, les étoiles sont souvent appelées génériquement des piliers : «Les piliers du ciel, Ruma, sont devenus de grandes étoiles scintillantes dans les cieux : Anâ-mua est le pilier d'entrée du dôme, Anâ-muri est le pilier pour noircir et tatouer, Anâ-roto est le pilier de la pureté parfaite » (Henry, 1993 : 44). En témoigne encore l'étoile Sirius nommée $T e$ $P o u$, le Poteau, à l'île de Pâques [information directe recueillie en 2001]. Car mer et ciel sont à l'origine intimement mêlés et, dans la mythologie, il avait fallu un énorme effort aux dieux $R \hat{u}$, Maui, et Tane pour les maintenir séparés par des poteaux, des piliers, et ainsi organiser le ciel en couches superposées, où circulent parmi les astres des dieux et des héros plus ou moins lointains. Les étoiles sont les sommets de ces piliers qui soutiennent le ciel, tandis que la base du pilier devient ce lieu précis sous le zénith de l'étoile, nommé le rua de l'étoile, qu'on pourrait traduire par 'sous le trou de l'étoile', ou 'l'autre bout de l'étoile', rua signifiant à la fois 'le trou' et 'le second, la paire'. Une île se trouvant dans le rua, sous le passage zénithal d'une étoile brillante, lui sera donc intimement associée comme l'autre extrémité du pilier. Le lien est si serré que nombreuses sont les îles qui sont désignées par le nom même de cette étoile. Je m'appuie ici sur le très riche travail de Guehennec (1985), présenté dans le dernier chapitre du Ciel de Tahiti et des mers du Sud de Maurice Graindorge où l'on trouve comme noms d'étoile: Pora-Pora (p. 138), nom donné à
Hawaii pour une étoile dans le centaure. Ainsi nommait-on l'île de Bora-Bora au $\mathrm{XIX}^{\mathrm{e}}$ siècle ; Kahikinui (p. 147), nom d'une autre étoile à Hawaii, qui donna d'évidence Tahiti Nui ; Fetu Hiva (p. 143) qui est à la fois une étoile et une île aux Marquises ; Mangaroa (p. 144), nom donné à la Voie Lactée à... Mangareva. Quand, d'après les indications de Guéhennec, j'ai pu localiser précisément ces étoiles, je me suis aperçue qu'il y avait presque à chaque fois coïncidence entre la latitude de l'étoile et celle de l'île.

Il y avait donc une correspondance serrée entre les îles et les étoiles. Non pas une superposition des cartes de l'océan et du ciel. Mais le ciel, en tournant au cours de la nuit, place à un moment donné l'étoile brillante et remarquable qui porte le nom de l'île juste au-dessus de celleci. Les étoiles de deux îles proches ne seront pas forcément visibles ensemble, et leur dispersion dans le ciel ne ressemblera pas à la dispersion des îles. Mais chaque étoile donnera exactement la latitude de l'île associée (Fig. 1). Ainsi le ciel pouvait effectivement être lu comme une carte en latitude du pays polynésien. Il pouvait permettre de situer immédiatement les îles sur une échelle allant du Nord au Sud, dans l'absolu et entre elles.

La relation serrée entre étoiles et îles, qui a souvent été mise en évidence dans les récits mythiques, existait donc aussi entre la géographie de la Polynésie et le ciel étoilé. Relation que je qualifierai de projection travaillée, installée dans le temps de la nuit et de l'année. Pour les Polynésiens, le ciel était comme un atlas perpétuellement ouvert de leur pays, dans sa vastitude et sa complexité, présentant ainsi toujours l'île où l'on était parmi les autres, les îles étant à la fois séparées et reliées entre elles - en tout cas jamais isolées - mais prises dans une vision globale. Quel que soit l'isolement du lieu de l'observation, la totalité des îles était lisible, grâce au ciel étoilé.

Cette vision céleste du pays polynésien pourrait peut-être expliquer d'autres « erreurs » de la carte de Tupaia, en fait des erreurs de longitude : quand Tupaia montre une étoile puis une autre, en nommant une île puis une autre, Cook n'a, semble-t-il, pas compris la relation qui s'établissait ainsi. Même si lui-même, navigateur, était très au fait de la difficulté de préciser la longitude d'un lieu, avait-il perçu que l'écartement entre les étoiles indiquées par Tupaia était peut-être complètement différent de l'écartement entre les îles nommées?

Un autre usage des étoiles en verticalité, plus prosaïque, est de pouvoir garder un cap, en observant l'alignement de certaines étoiles. Les 

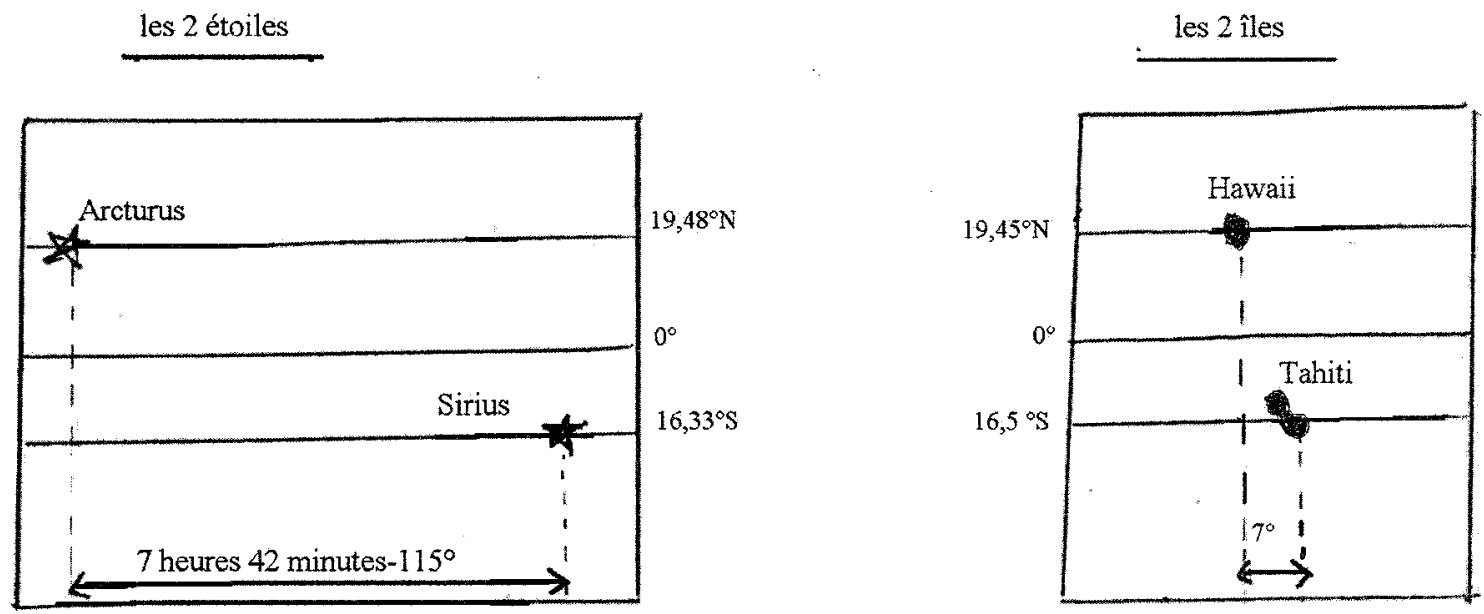

\author{
Position de Sirius et d'Arcturus et des dîles \\ associees, Tahiti et Hawaii. \\ Il y a bien correspondance en latitude, mais pas du tout \\ en "écartement".
}

FIG. 1. - Comparaison des écartements de deux étoiles et des deux îles correspondantes.

trois étoiles du Baudrier d'Orion montrent constamment la direction Est-Ouest dans leur parcours nocturne. Ces étoiles sont parfois nommées Na Tautoru en Polynésie, les Trois Étoiles, nom bien en résonance avec Les Trois Rois provençaux, ou Los Tres Maria des Espagnols, étoiles nommées parfois aussi Ta'urua o Mere Matu Tahi, Réjouissance des Désirs des Parents, appellation surprenante de grandeur et de chatoyance, comme le sont souvent les noms polynésiens des astres. On s'alignait sur cette direction selon un angle donné, car la pratique de lecture d'un angle était si acquise et si sûre qu'on pouvait le transposer mentalement à un tout autre endroit du ciel.

\section{Le ciel partout, pour tout}

Tout cela était complété par la perception globale de la voûte céleste, tellement intégrée qu'une seule petite partie aperçue suffisait pour avoir la vision mentale du tout. Le ciel était divisé en plusieurs régions. Dans la boussole d'étoiles reconstituée par Ben Finney, un grand oiseau, Manu, est étendu en travers du ciel, ailes déployées, de direction NNO-SSE (Fig. 2).

Cet oiseau divise l'horizon en quatre « maisons ", dit la traduction, c'est-à-dire en quadrants : celui correspondant au bec, à la queue, puis à l'aile droite, et à l'autre aile. Le corps est la maison centrale. Mais ailleurs, ce pouvait être un poisson, comme en Micronésie, la bouche à l'Est, la queue à l'Ouest. (Finney, 1999 : 96).

Cette connaissance du ciel était tellement inhérente à leur culture que les Polynésiens ne purent l'expliquer, quand on le leur demanda. Ou peut-être ne le voulurent-ils pas, ne souhaitant pas divulguer leur héritage dès les premiers temps d'une rencontre. Pour eux, ils étaient dans et sous le ciel, qui leur était familier et lisible depuis toujours. Chaque endroit présentait un paysage étoilé particulier : un alignement horizontal de deux étoiles précises signifiait qu'ils étaient chez eux. Si cet alignement penchait vers le Nord, c'est qu'ils étaient trop au Sud. Ou l'inverse. En latitude, ils n'étaient jamais perdus.

La connaissance des étoiles n'était pas seulement une somme de savoirs, mais une manière d'être au monde. Elle était l'instrument permanent pour se repérer dans l'espace, pour connaître la saison, la date, la houle, les vents, les vols d'oiseaux. Les étoiles, les astres, étaient omniprésents.

À Tahiti, l'année était divisée en deux périodes, selon la position des Pléïades, Matari'i, « les petits yeux », traduction souvent admise mais que Stéphane Jourdan propose, dans son article très riche et judicieux, de remplacer par «les petits repères » (Jourdan, 2000). Pléïades audessus (période d'abondance), Pléiades audessous (période de restriction). Les noms des mois étaient associés à l'étoile dominante du moment, celle qu'on voyait à l'Ouest juste avant 


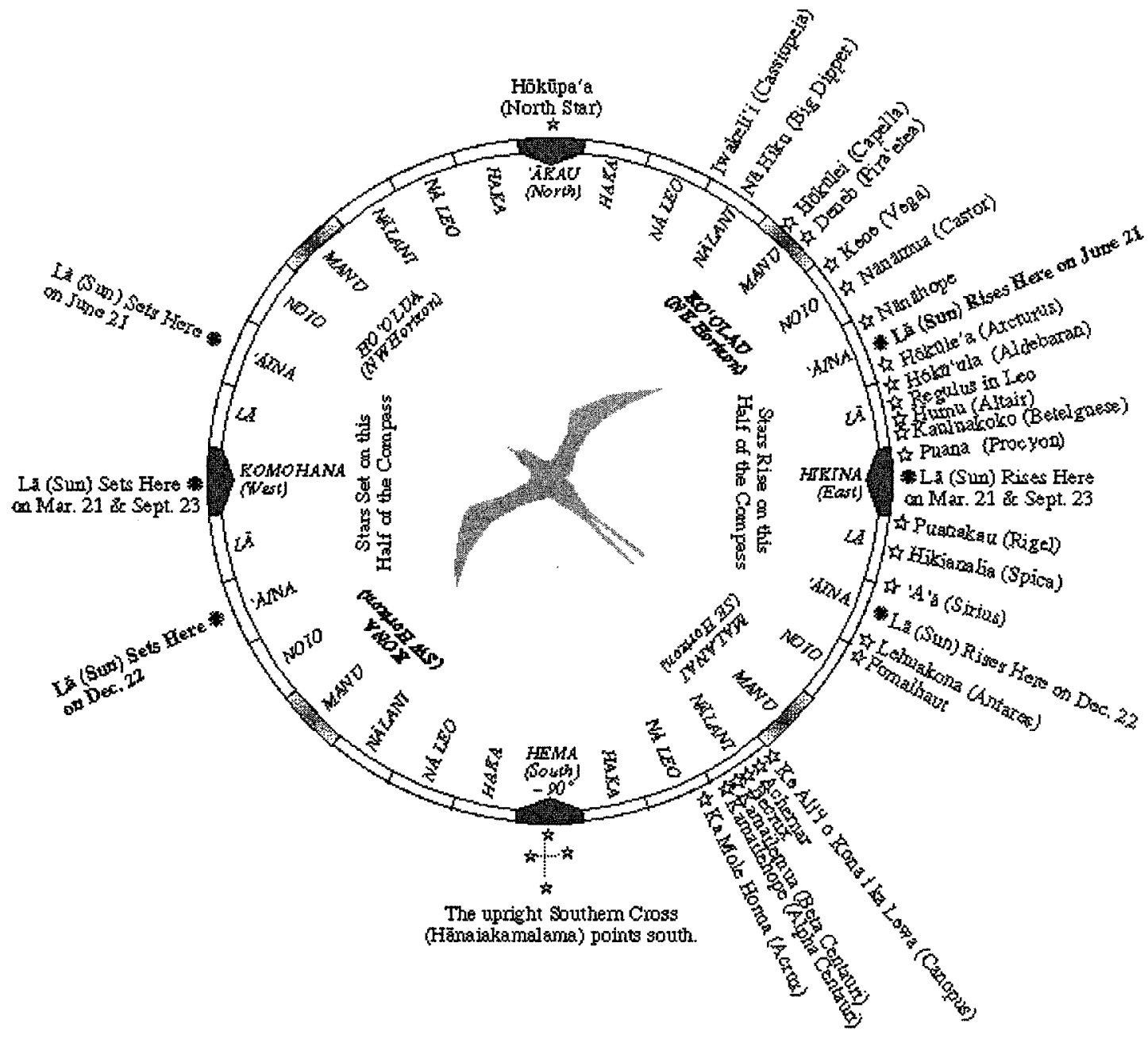

FIG. 2. - La boussole de 21 étoiles reconstituée par la Polynesian Voyaging Society [www.pvs.hawaii.org /navigate/BrightStars.html]

l'aube en regardant vers le haut, mais avant que le cou ne se plisse, c'est-à-dire autour de 45 degrés (Goodenough et Thomas : 1987) et qui descendait peu à peu au cours des nuits, jusqu'à disparaître, et qu'une autre étoile prenne le relais. Pipiri partout, Takurua (ou Kaulua, selon que l'on est aux Tuamotu ou à Hawaii), Herehu sont à la fois noms d'étoile et noms de mois.

Mais les noms des mois vont au cours du temps, au fur et à mesure de l'implantation durable dans des îles, au fur et à mesure de la précession des équinoxes aussi, qui fait inexorablement dévier le mois de son étoile, également refléter des préoccupations agricoles : le mois du ventre plein, le mois où il faut planter. Guehennec produit un calendrier recueilli auprès de la grande cheffesse de Papara en 1891, dans lequel à chaque mois est associée une épouse très terrestre, qui précise ou complète ses particularités. Ainsi le mois Pipiri (nom d'étoile, signifiant restriction), et son épouse, Pipiri-ma'ai-fenua, (restriction de nourriture dans le pays), ou le mois Varehu, printemps, et son épouse Oteatea, inflorescence de banane (Guehennec, 1985: 108).

Parallèlement, courait le mois lunaire, divisé en 29 ou 30 jours, ou plutôt en 29 ou 30 nuits, qui avaient toutes un nom particulier, souvent un nom décrivant l'apparence de l'astre : Omarae, Te Marama-Ati, (Pleine Face de Lune) à la pleine lune, Deuxième Jour de la Lune Pleine ensuite, ou Ore-Ore-Roto (rien-rien dedans) les nuits sans lune (Ellis,1972 : 77). Chacune de ces nuits avait des caractéristiques particulières : Tamatea, celle où l'on doit pêcher les grands poissons des profondeurs, la nuit Tane où l'on s'enlace pour engendrer de beaux enfants (Guehennec, 1985 : 105). 


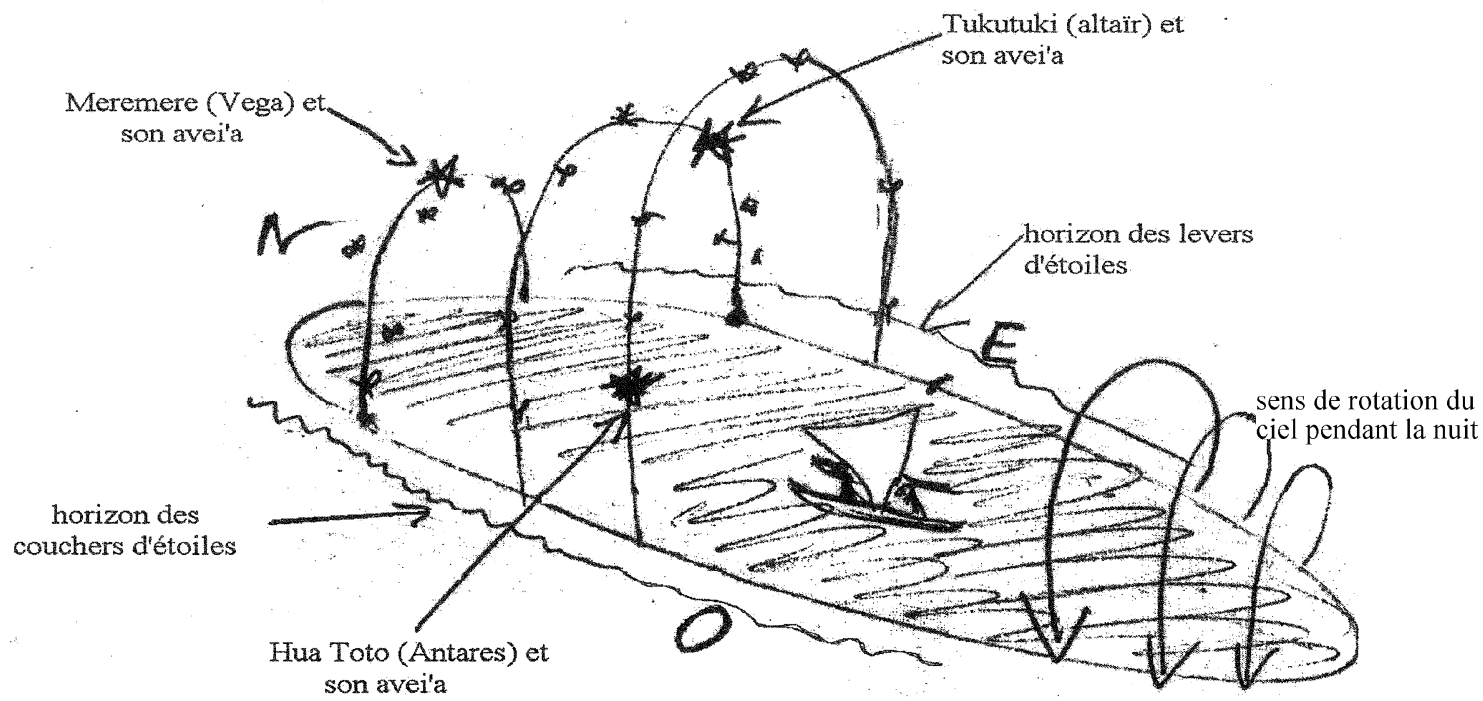

FIG. 3. - L'horizon ponctué des levers et couchers des étoiles principales et leur avei'a.

Les heures du jour reflétaient aussi l'attentive description de la nature, de la marée, (qui arrive à heure fixe à Tahiti, tout au long de l'année) et de la montée et descente du soleil. Voici la traduction de quelques-unes de ces heures du matin, données par William Ellis (Ellis, 1972 : 78) :

- l'heure du coq (aaoa te moa aaoa; aaoa est l'équivalent du cocorico)

- l'heure où les mouches commencent à bouger (ferao-rao)

- l'heure où l'on peut distinguer les traits d'un homme (itea te mata taata)

- l'apparition de la partie supérieure du soleil (te hatea ra o te ra)

- le lever du soleil (poi-poi)

- le soleil juste au-dessus de l'horizon (ofao tuna te ra)

- le soleil envoyant ses premiers rayons sur la terre (matiti titi te ra)

- midi, le zénith (avatea)

Celles du soir reflétaient la description de la descente de l'astre :

- déclin amorcé (hia hia te ra)

- déclin (ua maru maru te ra)

- coucher (ahiahi)

- tombée du soleil dans l'eau (mairi te ra)

- début de l'obscurité (arehurehu)

- la lumière complètement disparue ( $p o$ )

- la marée montante (pananu te tai)

\section{Étoiles à l'horizon : la boussole stellaire}

Pour la navigation, les Polynésiens utilisaient la particularité de ces latitudes où la Polaire est au Nord certes, mais à ras de terre ou de mer. Et où le ciel entier bascule durant la nuit d'Est en Ouest, comme si l'on était dans un cylindre. Les étoiles surgissent de la mer perpendiculairement à l'horizon, et se couchent pareillement, semblant plonger directement, sans l'effet tangentiel qu'il y a sous des latitudes plus élevées, comme en Europe (Fig. 3). Elles semblent toutes décrire un arc de cercle plus ou moins haut, se levant vers l'Est, se couchant symétriquement vers l'Ouest au cours de la nuit. Tout cela est fort bien décrit par David Lewis, dans son ouvrage We, the navigators (Lewis, 1972) : les 360 degrés de l'horizon étaient repérés par une véritable boussole stellaire, ponctuée de 32 repères, les points de lever ou de coucher des "étoiles-guides ». $\mathrm{Ce}$ pouvait être les mêmes étoiles que celles associées aux îles, mais dans une tout autre utilisation. Et toutes les étoiles se levant au même endroit qu'une étoile-guide étaient connues, apprises, reliées entre elles par ce qu'on appelait un "chemin d'étoiles", l'avei'a, en somme un arc de cercle d'une latitude. Les levers successifs des étoiles d'un même avei'a indiquaient donc une direction constante. Leur coucher pareillement.

Pour chaque destination, on indiquait la durée du voyage et la direction, donnée par une étoile-guide et tout l'avei'a associé, que devait pointer la proue du bateau. On donnait aussi l'étoile de la direction diamétralement opposée, qui devait rester dans l'axe de la poupe. Tenez, capitaine, le bateau entre ces deux repères. Et tenez compte du sillage pour la dérive... Était apprise aussi la paire d'étoiles à quatre-vingt-dix degrés, l'une à droite, l'autre à gauche de la direction générale. 


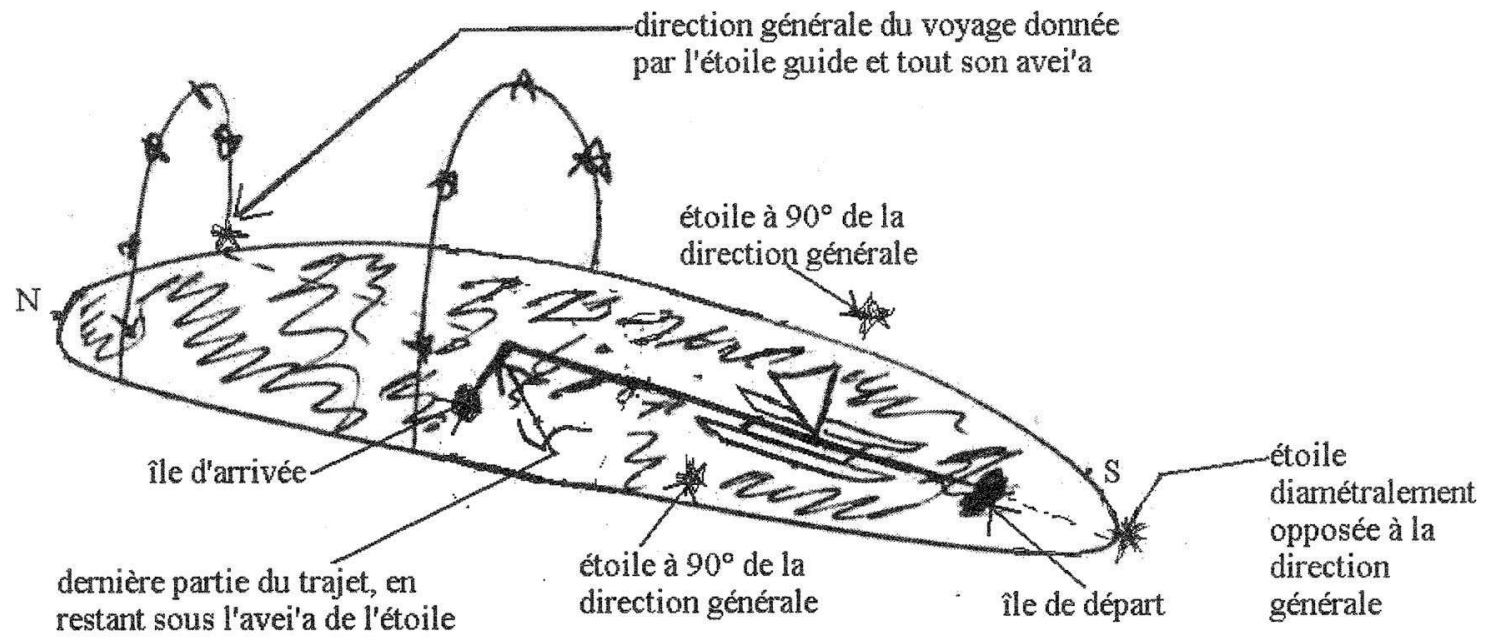

FIG. 4. - Voyage en « pique à $u r u$ ».

Il y avait donc pour une direction quatre repères lisibles dans quatre directions différentes à un instant donné, ce qui permettait de se diriger même si le ciel était en partie recouvert de nuages. La latitude finale du voyage était donnée par l'étoile "verticale" associée à l'île à atteindre. On cherchait à venir se situer au zénith de cette étoile, dans son rua, si possible un peu trop à l'est de l'île à atteindre. Puis on naviguait alors d'Est en Ouest en restant sous l'avei'a de cette latitude, porté par les vents dominants. Le voyage était ainsi formé de deux parties distinctes, un grand morceau droit pour atteindre la bonne latitude, suivi d'un autre à angle serré, pour, en restant sous cette latitude, trouver l'île recherchée. (Fig. 4)

\section{Etak}

La science de la navigation a perduré en Micronésie beaucoup plus longtemps qu'en Polynésie, et l'étude de la navigation micronésienne permet d'approcher ce que fut la navigation polynésienne, même si l'analogie complète ne peut être faite entre les deux cultures. David Lewis, Ben Finney, Ward H.Goodenough, Thomas Gladwin et Edwin Hutchins ont, par leurs recherches et leurs écrits, permis d'étudier cette navigation dans ses détails et particularités, comme l'etak.

L'etak, ce mystérieux procédé de navigation utilisé jusqu'à nos jours dans cette région du monde, pourrait être défini comme la méthode pour aller d'une île à une autre en suivant la progression du voyage dans les étoiles, grâce à une troisième île, associée en triangulation, île appelée lu pongank à Satawal (Finney, 1999: 111), signifiant « au milieu et en travers ». En fait, la réalité est beaucoup plus complexe. Si l'île associée est visible, le procédé est clair et compréhensible. Gladwin et Hutchins ont bien mis en évidence comment cette île était repérée glissant sur l'horizon d'un point de lever d'étoile à un autre (Fig. 5).

Effectivement, la lecture de la position de l'île à l'horizon, sur la boussole stellaire, permet une surveillance de la progression du voyage, qui est ainsi fractionné en etak, segments d'inégale longueur, déduits des étoiles associées. Avant de partir, on apprenait le nombre d'etak, et les particularités de chacun : celui où l'on croise le requin aux mouvements paresseux, l'etak de la raie, celui des oiseaux bruyants, celui où la houle a telle particularité...

Mais la singularité de cette méthode vient de ce qu'elle est aussi utilisée quand l'île associée est invisible, voire n'existe pas. Elle est alors inventée, sous forme d'un animal fabuleux, souvent une baleine immobile. C'est un fait courant dans la mythologie polynésienne, les îles sont souvent perçues comme des animaux marins, posés sur l'eau, flottants, et non des terres ancrées avec la mer autour. Une légende rapportée par Teuira Henry parle ainsi de Tahiti Le Poisson : Tahiti était une terre initialement rattachée à Hawaii. Une jeune fille transgressa un tabou qui interdisait de se déplacer et alla se baigner dans une rivière, dans un lieu appelé Opoa. Les dieux furieux la paralysèrent, et elle se noya. Une anguille avala son corps et devint possédée par l'esprit de la jeune fille. Enragée, elle arrachait les racines des arbres, la terre d'Opoa se détacha et devint un énorme poisson. Elle se dirigea vers 


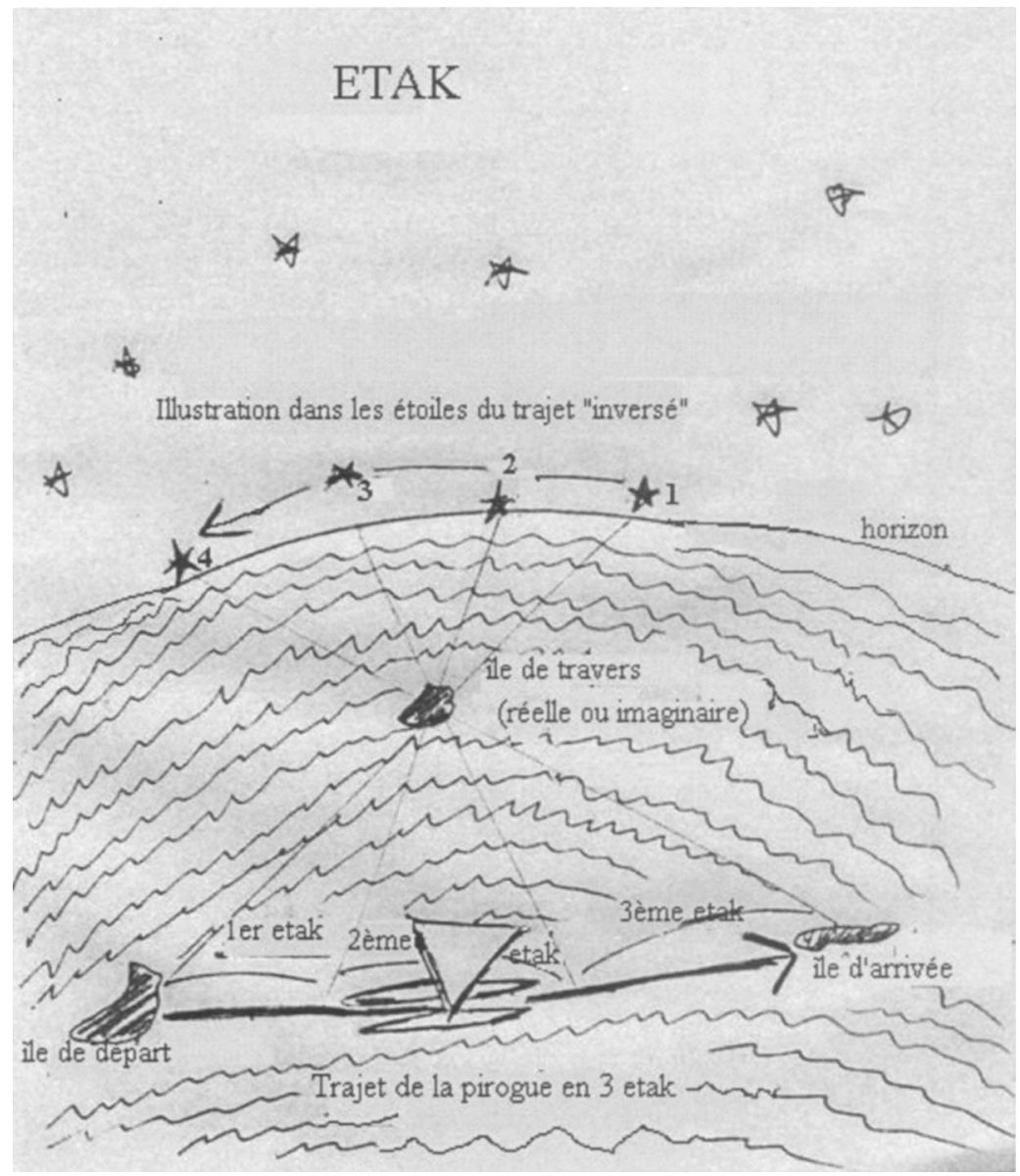

FIG. 5. - Voyage en trois etak.

« l'horizon d'un nouveau ciel » et là, fut stabilisée par le valeureux Tafa'i, qui coupa les tendons du poisson (Henry, 1993 : 102-104). On trouve aussi dans les îles de la Société une légende où le héros, Maui, pêcha une île qui se trouvait coincée sous la mer (Henry, 1993 : 95). Dans toutes ces légendes, l'élément majeur, l'espace dominant, est l'océan. Les îles ne sont que des balises, des havres, des ports, dans un espace de circulation.

Dans ce procédé de l'etak, une île-baleine fictive est donc posée sur le travers du trajet, et on imagine son glissement d'un point de l'horizon à un autre, d'un lever d'étoile à un autre. Là, il n'y a aucune vérification de la progression, puisqu'on ne voit pas l'île, mais bien plutôt une illustration dans les étoiles du trajet de la pirogue. Car, en Micronésie, la pirogue est perçue comme immobile, ce sont les îles - et donc les étoiles - qui se déplacent vers elle (Galdwin, 1970 : 181). Et comme l'a très finement analysé Hutchins, il faut abandonner aussi l'idée d'un horizon circulaire pour le remplacer par une ligne parallèle au trajet du bateau. Le glissement de la pirogue va être illustré par le maître de navigation en un trajet inversé dans les étoiles choisies, sur une ligne parallèle au bateau, mais dirigée dans le sens opposé.

$\mathrm{Si}$ le vent souffle bien, si la houle est porteuse, si l'eau file vite en chuintant sous le bateau, le maître traduira cela par le passage rapide de l'île invisible de l'étoile 1 à l'étoile 2 . Si les conditions sont moins favorables ensuite, il prolongera l'etak suivant, de l'étoile 2 à l'étoile 3 . De la surveillance de la progression de la pirogue quand l'île de travers est visible, l'etak devient donc, quand l'île est invisible, une illustration, une transcription au jour le jour du voyage, voyage qui s'inscrit, en même temps qu'il se fait sur l'eau, dans les levers et couchers des étoiles à l'horizon, visibles sur le côté. Se retrouve ici, comme dans le cas de l'atlas lisible aux cieux, une représentation dans les étoiles d'une réalité humaine, terrestre, marine, celle du voyage en pirogue. Le trajet des hommes sur l'océan a un écho en symétrie inversée dans les étoiles du ciel, étoiles qui permettent d'inscrire, et de « lire » ce 
trajet. Navigation aux cieux, inhabituelle, mais qu'on retrouve dans la mythologie, quand Tane, le grand dieu polynésien, vola une pirogue : « il la jeta dans son ciel, le dixième ciel, et Tane vogua sur sa pirogue dans son ciel clair et ouvert» (Henry, 1993 : 122).

Les levers et les couchers successifs de ce point imaginaire, fixe sur le côté, étaient appris avant de partir. Pareillement apprises les particularités des etak, ces « trajets de mer».

Pour fixer ces repérages, il y avait des chants dont les paroles étaient autant de repères mnémotechniques. Ces repères prenaient parfois l'apparence d'animaux réels, dauphins, baleines, oiseaux ayant l'habitude de fréquenter ces parages. Les marins ne les cherchaient pas systématiquement, mais leur rencontre éventuelle les assurait d'être sur le bon chemin. Certains chants évoquent aussi des animaux fabuleux, comme une raie à points rouges, un requin particulier, un poisson géant. D'autres illustrent une succession de vagues présentant chacune des épreuves à surmonter (Goodenough et Thomas, 1987: 188). Le profane pouvait n'y rien entendre d'autre qu'une fable marine. Le maitre révélait peu à peu le sens caché de ces paroles à son élève, jusqu'à ce que ce dernier parvienne à mémoriser toutes les bornes du parcours.

L'élève, devenu maître à son tour, personnalisait son récit, qui pouvait différer de celui d'origine, l'essentiel étant que tous les éléments du parcours soient présents, et décryptables. Chaque savoir était ainsi maintenu vivant, irrigué par celui qui le transmettait.

Ces chants pouvaient aussi aider d'une manière beaucoup plus subjective, proche de la transe chamanique à laquelle le chef de pirogue avait recours quand, par exemple, il était perdu en mer. Il les chantait jusqu'à ce que la litanie lui permette de réorganiser le schéma de sa route, autrement dit, de se calmer et de ne pas céder à la panique, pour retrouver la cohérence du chemin à suivre.

Dans son article "Bathymétrie légendaire" (Dunis, 1999), Serge Dunis rappelle certaines légendes polynésiennes, comme celle du voyage du géant Rata, et met en évidence les trajets qu'elles décrivent. Pour Rata, c'est de Tahiti à Pitcairn. Hono'ura, autre géant, fit lui route de Bora-Bora à Napuka. Tafa'i, géant lui aussi, vécut des péripéties qui nous promènent de Tahiti à Hawaii. Ces légendes, de par les trajets maritimes qu'elles décrivent, ne seraient-elles pas issues d'anciens chants de navigation?

L'etak est donc tout ce système complexe à la fois très abstrait et très concrétisé, permettant de relier un voyage en pirogue aux étoiles et aux particularités marines locales, fait d'un savoir objectif et aussi de perceptions surnaturelles, science et chansons intimement mêlées, les chants illustrant la science, la science suscitant de nouveaux chants. Après le contact avec les Européens, d'autres horizons s'étant ouverts, il y eut création de nouveaux parcours mentaux et de nouvelles récitations apprises et chantées.

\section{Naviguer aussi sans étoiles}

Aussi ingénieuse, complexe, précise et efficace que soit la connaissance des étoiles, elle ne servait en mer qu'une partie du temps. Certes, les courts trajets se faisaient de préférence les nuits étoilées. D'ailleurs, la distance entre deux îles est aujourd'hui encore indiquée à Tahiti en durée de parcours commençant par la nuit : « Tahaa est à une nuit, un jour, et encore la moitié d'une nuit de Tahiti en pirogue ", m'indiquait un voisin à Faaone. Mais d'autres pans du savoir de la navigation permettaient aussi de suivre sa route le jour, ou lors des nuits sans étoiles.

Autre source d'information, la lecture de la houle. Elle avait des noms différents à Satawal, aux Carolines rilib, kaelib, bungdockerik, bungdockeing (Goodenough, 1987) qu'on reconnaissait selon le bruit que faisaient les vagues en frappant la coque, ou selon l'intervalle de temps entre deux vagues, ou selon leur couleur, différente selon les vents. Les meilleurs pilotes les identifiaient même dans le noir, à leur manière de passer sous le bateau. La houle dominante avait une direction constante connue, sur de larges zones d'océan. Le bateau était soigneusement orienté au lever du soleil. Soleil qui se levait, on le savait bien, non pas à l'Est mais, suivant la saison, à un point précis entre Nord-Est et Sud-Est. À ses premières lueurs, on observait attentivement les premières vagues, pour en connaître la direction et fixer le cap. Pareil à son coucher. Il y avait les zones de houles contrariées, dues à la présence des terres plus ou moins lointaines, où les vagues s'éclatent l'une contre l'autre, générant des éclaboussures blanches. Ces zones de crêtes écumantes étaient perçues comme de grands sentiers balisés, qu'il suffisait de suivre.

Illustrent ce savoir les cartes des îles Marshall, en Micronésie, faites d'entrelacs de bois légers, ponctuées de coquillage. Les cartes rebbilib, cartes d'océan, riches d'indices (Fig. 6) : la direction de la houle montrée par des baguettes parallèles, les courants déviants par des lianes souples, les zones de houles contrariées s'y lisent clairement, et les îles bien sûr, représentées par les coquillages (Siorat, 1980). 


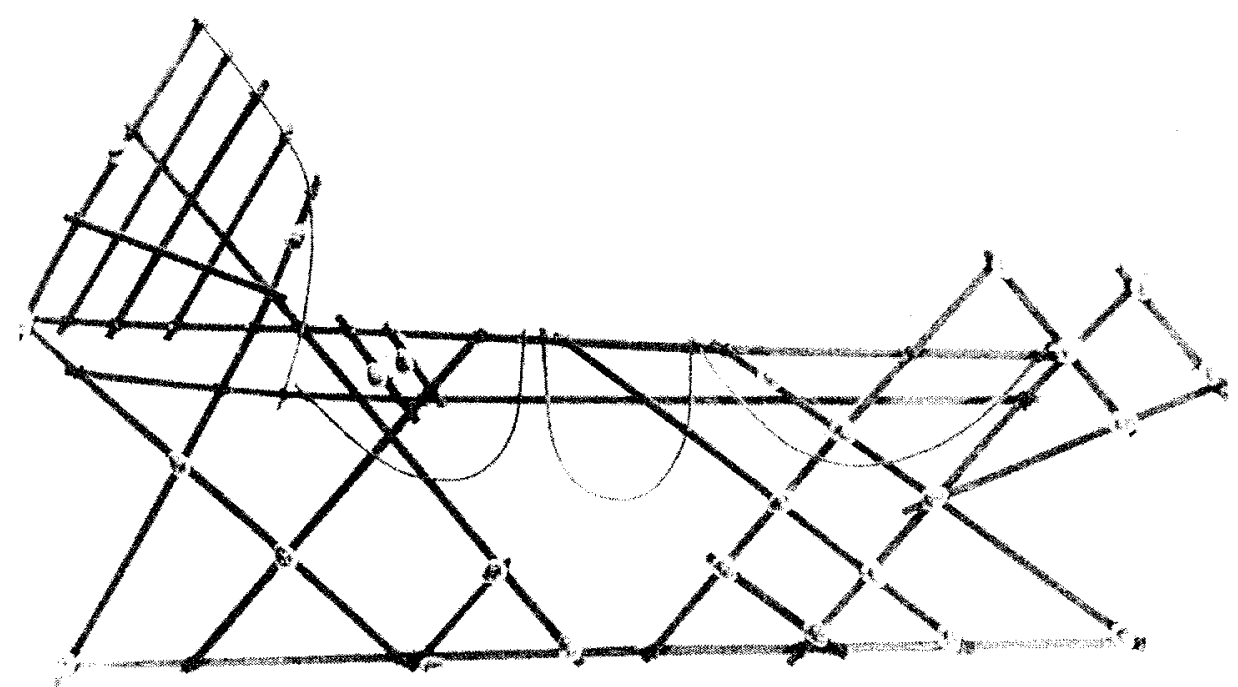

Fig. 6. - Carte rebbelibs, carte d'océan des îles Marshall en baguettes de bois, fibres et coquillages (Photo Musée de l'Homme, cliché J. Oster).

Ces cartes avaient une grande valeur. Le maître de la pirogue s'en imprégnait avant le voyage, une fois la route choisie, mais jamais elles n'étaient consultées en chemin. Il en allait de l'honneur du maître, qui devait les avoir parfaitement mémorisées avant de partir. Elles se transmettaient de père en fils, ou en fille. Certaines autres étaient des cartes d'école (cartes mattang) pour illustrer et comprendre la lecture des houles, justement. D'autres encore (cartes meddo), un mélange des deux. On ne sait pas si de telles cartes existaient en Polynésie, où les îles hautes sont plus fréquentes, donc plus facilement repérables. Mais on peut supposer qu'un support, fonctionnant sur le même principe, a pu être utilisé.

Doublant la boussole d'étoiles, une sorte de rose des vents indiquait le nom, la direction et la saison des vents dominants, désignés, on l'a vu dans la carte de Tupaia, par là où ils menaient. Ces vents sont connus pour certains encore aujourd'hui, comme le mara'amu à Tahiti, vent froid d'hiver, venant des zones glacées du Sud. Le jour, ils donnaient aussi une information sur l'orientation du bateau.

Existaient aussi pour la navigation de nuit de fantastiques, simples et étranges «boussoles éoliennes » faites d'une planche où l'on fixait deux noix de cocos percées, chacune émettant au vent un son propre et reconnaissable (Fig. 7). Elles étaient orientées dans deux directions différentes, si bien qu'une seule noix sifflait à la fois. La nuit, un changement du sifflement indiquait donc un changement de cap, qu'il fallait rectifier (Neyret, $1966: 26$ ). L'existence de ces boussoles a parfois été mise en doute, mais Éric Conte
(1986) affirme avoir rencontrer des pêcheurs de Napuka qui les avaient eux-mêmes utilisées.

Autre procédé étonnant: pour savoir si la zone d'un courant plus chaud était atteinte, on se plongeait le derrière, et lui seulement, dans la mer, certaines zones sensibles du postérieur étant de bien subtils détecteurs de variation de température (Lewis, 1974 : 752). Suivre un courant se disait « naviguer sur le dos d'un courant », qui vous portait comme une bête docile.

\section{S’approcher}

Les étoiles, la houle, les vents et les grands courants permettaient donc de suivre une direction pour une approche globale de la zone d'une île. Pour la trouver exactement, il y avait toute une technique d'approche qui permettait d'entourer chaque île d'un halo d'une centaine de kilomètres. Comme les îles s'organisent souvent en archipel, ce grossissement permettait d'en trouver une en visant dans le tas. Pas toujours la bonne, mais arrivé là, on obtenait des habitants les renseignements pour trouver l'île cherchée. Pour des îles isolées comme l'île de Pâques, la difficulté était plus grande.

Premier renseignement d'approche dont parle Lewis, très intrigant et mal connu, visible à plus de cent kilomètres, en particulier les nuits noires et pluvieuses, les éclairs d'une foudre sousmarine, montrant le chemin vers l'île, en profondeur. Cela pourrait être lié au mouvement sousmarin des houles profondes, contrariées par une terre. Ce phénomène s'estompe lorsqu'on s'en approche plus près, mais il est relayé par le chan- 


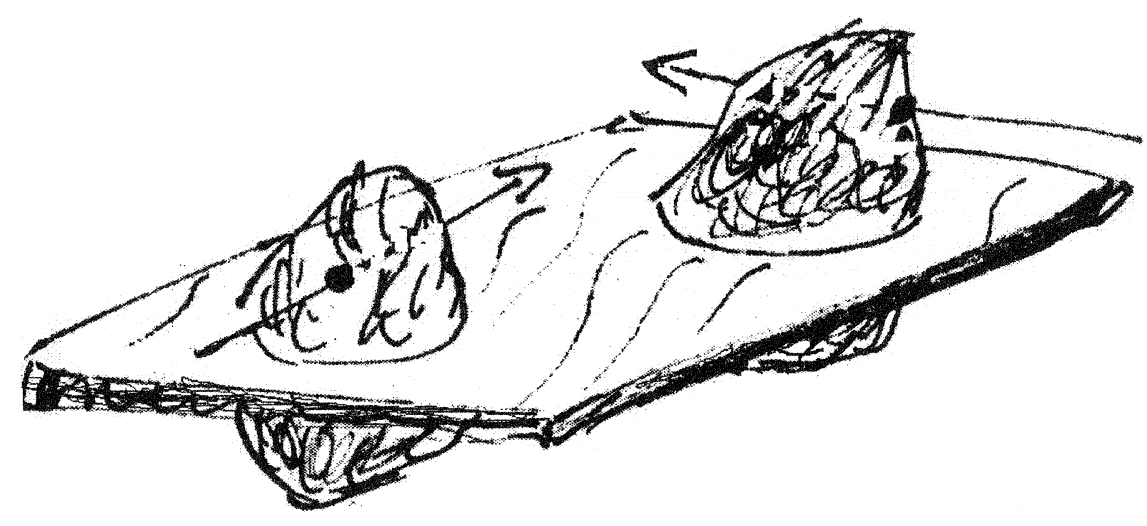

FIG. 7 : Boussole éolienne

gement de la houle de surface qui, à environ cinquante kilomètres de l'île, se "plisse » différemment. Le bateau change alors de tangage.

Puis les oiseaux. La présence des fous, puis les sternes, puis les noddis indiquaient qu'on se rapprochait, et leur premier vol du matin montrait la direction précise de la terre (ils venaient le matin de l'île, et y retournaient le soir). Mau Pialug était ainsi à l'aube sur un bateau, guettant les premiers oiseaux d'une île que l'équipage situait au Sud. Mais les oiseaux arrivèrent du Nord. Ses compagnons, pensant qu'ils avaient dépassé l'île durant la nuit, voulurent faire demitour, quand Mau leur dit de n'en rien faire. Un des oiseaux, avait-il vu, avait un poisson dans le bec. Il revenait nourrir ses petits. C'était la saison des nichées durant laquelle les oiseaux partent deux fois en mer, une fois avant le jour, pour les oisillons, puis une autre fois pour eux-mêmes. Effectivement, une heure plus tard, les oiseaux réapparurent dans le bon sens. Silence, admiration. Certains ont le savoir, d'autres doivent écouter encore (Thompson, 1980).

La formation des nuages à l'aplomb d'une île, leur forme et leur couleur étaient également des phénomènes connus. Nuages immobiles, vissés sur une terre comme chapeaux sur la tête. Ils diffèrent selon qu'on approche d'une île haute ou d'un atoll. Les gens de mer parlent encore aujourd'hui à Tahiti du reflet vert du lagon de l'atoll d'Anaa visible dans les nuages.

On observait aussi les débris végétaux flottant sur l'eau, et leur degré de décomposition.

Pour finir, sachant l'île proche, on jetait à l'eau des animaux, des cochons, ou des chiens, qui d'instinct nageaient vers la terre, même les lézards, m'a-t-on dit (témoignage direct d'un Marquisien recueilli en 2001).

\section{Apprendre}

Toute cette science de navigation était transmise à un groupe d'initiés, soigneusement éduqués. L'apprentissage durait longtemps, de six ou sept ans à l'âge adulte, et ce n'est que vers dix-huit ou vingt ans qu'un jeune se voyait confié sa première pirogue. Les élèves apprenaient par cœur les noms des étoiles-guides, et tous les avei'a, les chemins associés, autant d'arcs de cercles lisibles dans le ciel pour qui savait y voir, comme l'est encore chez nous l'écliptique. Ils apprenaient les couples des étoiles associées, diamétralement opposées.

Par cœur les litanies d'un parcours donné, avec les repères en triangle, avec les particularités de l'océan tout au long de la route, jalonnées d'amers. Par cour les noms des houles, des vents. Des exercices précis leur étaient demandés. Ainsi, rapporte Thomas, en Micronésie, le jeu «sonder le trou du récif» qui retrace le voyage virtuel d'un poisson-perroquet qui, délogé, part se réfugier dans une autre île, île dont on devait vite indiquer la route. Le jeu de " la pique à uru » qui consiste à se placer mentalement sur une île et à réciter les parcours pour, de celle-ci, joindre toutes les îles aux alentours, comme une pique à uru tourne sur elle-même pour détacher les fruits. On dessinait sur le sable des cartes marines avec des brindilles, des coraux et des coquillages, certains représentant des îles et d'autres des étoiles.

\section{Nommer}

Les Polynésiens nouvellement débarqués recréaient souvent une toponymie liée à l'île 
d'origine. Ainsi retrouve-t-on, sur des îles très éloignées, des noms de lieux semblables : à Rapa Nui et à Tahiti, deux lieux nommés Tautira. Deux autres Taravao, l'un à Hawaii, l'autre à Tahiti. Aux îles Cook et à l'île de Pâques, deux endroits nommés Poike. Mais les noms des lieux sont donnés dans la continuité de l'histoire, et deux lieux homonymes n'auront pas la même résonance car le nom d'un même personnage se verra chargé de significations nouvelles dans une île récemment colonisée.

Cette façon de porter ainsi le nom - à défaut de sa maison - sur son dos a sans doute grandement contribué à préserver la cohérence d'un peuple malgré le grand éloignement géographique, la toponymie familière reproduisant un univers connu, sinon un paysage, sur des îles liées beaucoup plus que séparées par la mer. Comme le montre bien Stéphane Jourdan, il semble qu'il y ait ainsi en Polynésie un effet ping-pong entre les lieux, les héros et les étoiles, un héros donnant son nom à une étoile, elle-même donnant le nom à une île, et un lieu particulier de l'île étant de nouveau consacré au dieu. "C'étaient tous des personnages royaux à $\mathrm{Fa}$ 'a-hiti depuis la période des ténèbres, et chacun avait son étoile. Ils portaient le nom de ces étoiles et les noms ont été perpétués dans les marae de ce monde » (Henry, 1993 : 46). Ainsi, Make Make est-il le nom d'un dieu à l'île de Pâques et d'un quartier à Atuona, aux Marquises. Rehua est l'étoile Antarès en Nouvelle-Zélande et aussi le nom d'un dieu plein de bonté, dissipant les chagrins et les peines, dans le panthéon maori. Car sur la mer et dans le ciel, espaces intimement mêlés, circulaient à l'origine les dieux et les héros, les fondateurs d'une lignée, souvent un couple incestueux lié au soleil, à la lune et aux étoiles.

Restent parfois au ciel des traces de ces parcours, dans le nom des astres: par exemple l'Hameçon de Maui, le héros qui pêcha la terre (c'est la même constellation que la queue du Scorpion occidental, mais présentée à l'envers, inversion des hémisphères oblige). $\mathrm{Ou}$ les Pipiri-ma, un frère et une sœur enfuis au ciel car mécontents, insatisfaits, leurs parents ne leur présentant pas assez vite les poissons qu'ils avaient pêchés. D'autres traces du parcours des dieux-héros apparaissent dans les noms d'îles où ils sont arrivés et ont fait souche. Le nom d'Hotu Hiva, une princesse de Huahine, se retrouve en partie dans les Hiva des îles marquisiennes (Nuku Hiva et Hiva Oa). Faisait-elle partie du premier voyage? Une île d'Hawaii se nomme Maui, comme le héros pêcheur d'îles.

\section{Conclure}

$\mathrm{Ne}$ retenir des étoiles en Polynésie que la notion de boussole stellaire, procédé de navigation, est aussi réducteur que de restreindre la science de la navigation polynésienne aux seules étoiles. Ce sont deux pans du savoir polynésien, certes en correspondance, mais tous deux infiniment vastes, irrigués par l'humain, baignés par toute une mythologie. Ce sont deux fragments d'une culture qui doivent être replacés dans la perception globale que les Polynésiens ont de l'Univers.

La navigation fait appel à une somme de connaissances précises, mais aussi à une capacité à atteindre un état de réceptivité tel que les multiples signes de la mer et du ciel deviennent lisibles et décryptables. Le maître de la pirogue, navigateur, astronome, mais aussi chaman devait, rapporte Nainoa Thompson, faire corps au plus près avec son bateau, chantant, dansant le voyage, se couchant parfois de tout son long sur le pont pour mieux sentir la course de la pirogue, et l'interpréter.

Les astres étaient certes des repères très bien maîtrisés pour la navigation, mais faisaient, font encore partie d'un ensemble plus vaste, lié à toute une astronomie-cosmologie. Ils restent un élément fondamental de cohérence du peuple polynésien, liant en un Tout la mer, le ciel, les îles, les étoiles, les lieux, les héros et l'année. Ils permettent aussi de se repérer dans ce grand pays de petites terres si dispersées, de s'y mouvoir, de s'y inscrire, comme j'ai tenté de le montrer, quand le ciel est lu comme un atlas, ou comme une carte de « chemins de mer».

Mais les étoiles polynésiennes renvoient encore à d'autres aspects : elles étaient aussi souvent liées à un héros, ce qui leur conférait un caractère subjectif, caractère utilisé aussi en navigation en tant que tel (comme l'étoile Arcturus, appelée à Tahiti 'Anaa Tahu'a a Ta'ata Metua te Tupu Mavae, Le Prêtre Bienveillant qui Grandit dans l'Espace, à laquelle certains préféreront se référer plutôt qu'à Hua Toto, la Vulve Rouge Antarès - pour des raisons toutes personnelles). Elles avaient toutes en général des noms favorables. Partir pour naviguer sous Mere, l'Amour des Parents, ou sous Vai Ora, l'Eau qui Donne la Vie, ou guidé par Ta'urua-e-Hiti-i-Ara-o-teAnuanua, Festivité Qui Montre Le Chemin De l'Arc-en-Ciel, ou encore 'Aana Tahu'a Vahine o Toa te Manava, la Prêtresse au Cour Brave, la route s'annonçait déjà belle. Belle comme l'est toute cette aventure du peuplement du Pacifique Est : audacieuse, mais expérimentée, et inscrivant au fur et à mesure son histoire dans les cieux. 


\section{RÉFÉRENCES}

CONTE, Éric, 1986. La navigation hauturière : un art disparu in Bernard Salvat, Encyclopédie de la Polynésie, vol 3, Tahiti, C. Gleizal/Multipress, pp. 54-56.

DunIs, Serge, 1999. Bathymétrie légendaire in Serge Dunis (dir.), D'île en île Pacifique, Paris, Klincksieck, pp. 127-169.

Ellis, William, 1972. À la recherche de la Polynésie d'autrefois, Société des Océanistes $n^{\circ} 25$, Paris, Musée de l'Homme.

FinNey, Ben, 1999. Calcul mental hauturier in Serge Dunis (dir.), D' île en île Pacifique, Paris, Klincksieck, pp. 91-119.

GLADwin, Thomas, 1970. East is a big bird. Navigation on Pulluat atoll, Cambridge, Massachusetts University Press.

Goodenough, Ward H. and Steve thomas, 1987. Traditional Navigation in Western Pacific, Expedition vol $29, \mathrm{n}^{\circ} 3$.

GueHENnEC, Constant, 1985. L'Année et le ciel polynésien dans les îles de la Société d'avant le contact, in Maurice Graindorge, Le ciel de Tahiti et des mers $d u$ Sud, Tahiti, Haere Po No, pp. 96-159.
Henry, Teuira, 1993 [Hawaii, 1928]. Mythes Tahitiens, Paris, Gallimard.

Hutchins, Edwin, 1983. Understanding Micronesian Navigation in D. Gentner and A. L Stevens (eds.), Mental Models, Hillsdale, Laurence Erlbaum Associates, pp. 191-225.

Jourdan, Stéphane, 2000. La toponymie aux îles Marquises, Te RORI [site internet: tahitinui.ifrance.com/tahitinui/rori.htlm].

Lewis, David, 1972. We, the navigators, Honolulu, University Press of Hawaï.

- 1974. Wind, wave, star and bird, National Geographic Magazine, Décembre 1974, pp. 746-763.

Neyret, S.M, 1966. Pirogues océaniennes, Triton $\mathrm{n}^{\circ} 76$, supplément de Neptunia $\mathrm{n}^{\circ} 81$, Paris, Les Amis du Musée de la Marine, pp. 21-28.

Siorat, Jean-Paul, 1980. Entre houle et île, Cartes et Figures de la terre, Paris, Centre Georges Pompidou, pp. 231-235.

Thompson, Nainoa, 1980. Finding a Way: 19741980, Polynesian Voyaging Society [site internet: pvs.hawaii.org/nainoa80tahiti.htlm]. 